\title{
Multimodality Cardiac Imaging - a Complex Interdisciplinary Approach Resulting from the CARDIO IMAGE Project
}

\author{
Theodora Benedek ${ }^{1,2}$, Imre Benedek $k^{1,2}$ \\ 1 University of Medicine and Pharmacy, Tîrgu Mureș, Romania \\ 2 Center of Advanced Research in Multimodality Cardiac Imaging, CardioMed Medical Center, Tîrgu Mureș, Romania
}

Given the multitude of complex and reliable information provided in different clinical settings, cardiac imaging became nowadays an indispensable tool for clinical practitioners and for clinical researchers as well. Invasive and noninvasive technologies have been developed in order to provide the clinicians with the necessary information on cardiovascular structure and function, required for choosing the best therapeutic approach in patients with heart diseases. In line with the modern technological developments, sophisticated imaging methods were implemented in the recent years, able to offer a more complex panel of data regarding heart structures and vascular system, methods which have been validated as cutting-edge technologies and golden-standard techniques, not only for clinical routine, but also for research applications.

Invasive cardiac imaging is a tool mainly dedicated to the assessment of coronary vessels during coronary angiography or coronary revascularization procedures. Apart from the classical coronary angiography, such modern invasive imaging tools include optical coherence tomography (OCT), intravascular ultrasound (IVUS) and nearinfrared spectroscopy (NIRS), all of them being frequently used not only for assessment of the severity of a coronary stenosis, but also for estimation of plaque vulnerability, es-

Theodora Benedek • Str. Gheorghe Marinescu nr. 38, 540139 Tîrgu Mureș, Romania Tel: +40 265215 551. E-mail: theodora.benedek@gmail.com

Imre Benedek • Str. Gheorghe Marinescu nr. 38, 540139 Tîrgu Mureș, Romania.

Tel: +40 265215 551. E-mail: imrebenedek@yahoo.com pecially in acute settings. However, their role in the evaluation of coronary lesions started to decline in parallel with the rapid development of noninvasive techniques for the visualization of coronary plaques, represented mainly by cardiac computed tomography angiography (CCTA). Being able to provide accurate data regarding stenosis severity and plaque structure and vulnerability, this technique replaced nowadays not only the invasive intracoronary techniques, but also the coronary angiography as a routine method for the visualization of the coronary tree.

However, despite being extremely useful for the estimation of the severity of coronary artery diseases on a large scale, none of the above mentioned methods is able to provide a simple information, which is critical for the therapeutic decision, represented by the functional significance of a coronary stenosis. Therefore, new techniques have been developed in order to offer a complex multimodality approach that would be able to answer this question.

The functional significance of a coronary stenosis can be assessed nowadays directly or indirectly, using invasive or noninvasive tools. Invasive determination of the fractional flow reserve (FFR) has been validated as a gold-standard technique for the estimation of the functional impact of a coronary stenosis, serving as a useful clinical decision tool. The invasive evaluation of FFR tends to be replaced by noninvasive evaluation using the so-called FFRCT, which implies the determination of the fractional flow reserve remotely, noninvasively, using computational flow dynamics for simulations of the coronary flow. Other imaging-based 
techniques proposed for assessing functional severity of a coronary artery stenosis are represented by transluminal attenuation gradient on CCTA, assessment of myocardial perfusion using computed tomography, echocardiography, MRI, or even a hybrid approach based on multimodality imaging.

A nice series of review articles and clinical updates published in this number of JIM, acknowledging the role of the CARDIO IMAGE project, provides an integrative approach on the role of modern cardiac imaging techniques for assessing coronary plaques, their hemodynamic impact and application in different clinical settings.

An article published by Nyulas et al. in this number presents the main CT features associated with coronary plaque vulnerability and the role of CCTA in detecting high-risk coronary plaques that are prone to rupture and can result in an acute coronary syndrome. ${ }^{1}$ However, the description of morphological features associated with vulnerable plaques cannot serve as a decision tool in the absence of clear data regarding their hemodynamic impact, which is the key determinant of myocardial ischemia.

Two reviews published in this number, by Chițu et al. and by Mester $e t$ al., address the topic of FFR assessment of functional significance as an extremely useful decision tool that can provide a solid basis for choosing the optimum therapeutic strategy.,2,3 Obtained either invasively using intracoronary probes or noninvasively using CTderived data and computational fluid dynamics, FFR has a major role in the therapeutic algorithm of patients with complex coronary artery diseases, and the role of modern technologies in obtaining this crucial information is nicely described in the two reviews, proving that FFR has a distinct role in the multimodality imaging approach of the patient with coronary artery disease.

Another clinical update published by Orzan et al. describes another technology that would be as reliable as FFR in assessing the hemodynamic significance of a coronary stenosis. ${ }^{4}$ This is represented by the Transluminal Attenuation Gradient, determined by CCTA, a new imaging-based biomarker characterizing coronary stenoses. However, the role of this new biomarker as an alternative to invasive or noninvasive FFR still needs to be validated in different clinical settings.
The functional significance of a coronary stenosis is not limited to the transluminal gradient or FFR. Two reviews published in this number by Stănescu et al. and Jáni et al. address the topic of myocardial perfusion, myocardial ischemia and myocardial viability as relevant features reflecting the severity of ischemia that results from a coronary stenosis or occlusion. ${ }^{5,6} \mathrm{An}$ interesting approach is the one related to hybrid imaging for the assessment of myocardial ischemia and viability, which proves that the current technological developments resulted in a significant shift from conventional imaging techniques to more and more sophisticated ones. In the future, these hybrid approaches could replace the traditional ones, at least in cutting-edge frontier research. ${ }^{5}$

Finally, the role of magnetic resonance imaging in the complex milieu of multimodality cardiac imaging is nicely addressed by Kovács et al. in the clinical update related to the role of atrial fibrosis in atrial fibrillation. This update reveals the role of cardiac imaging not only from the perspective of MRI assessment of cardiac fibrosis, but also from the perspective of complex 3D imaging in performing comprehensive ablation procedures based on intracardiac navigation with 3D imaging guidance. ${ }^{7}$

These seven articles underline the role of multimodality cardiac imaging as a complex interdisciplinary tool, at the border between cardiology, radiology and computer modeling and its major utility in cardiac care and in cardiologyoriented research.

\section{REFERENCES}

1. Nyulas T, Chițu M, Mester A, et al. Computed Tomography Biomarkers of Vulnerable Coronary Plaques. Journal of Interdisciplinary Medicine. 2016:1:263-266

2. Chițu M, Rat N, Opincariu D, et al. Invasive FFR - Current Applications and New Developments. Journal of Interdisciplinary Medicine. 2016;1:231-236.

3. Mester A, Chițu M, Rat N, et al. CT Determination of Fractional Flow Reserve in Coronary Lesions. Journal of Interdisciplinary Medicine. 2016;1:237-241.

4. Orzan M, Stănescu A, Nyulas T, et al. Transluminal Attenuation Gradient for the Noninvasive Assessment of Functional Significance in Coronary Artery Stenoses. Journal of Interdisciplinary Medicine. 2016;1:267-270.

5. Stănescu A, Opincariu D, Rat N, et al. Hybrid Imaging in the Assessment of Myocardial Ischemia and Viability. Journal of Interdisciplinary Medicine. 2016;1:242-246

6. Jáni L, Bordi L, Morariu M, et al. Imaging Techniques for the Assessment of Myocardial Perfusion. Journal of Interdisciplinary Medicine. 2016;1:247-251.

7. Kovács I, Mester A, Lehel B, et al. Myocardial Fibrosis and the Risk of Recurrence in Atrial Fibrillation. Journal of Interdisciplinary Medicine. 2016;1:259-262. 